\title{
LDL receptor-related protein mediates cell-surface clustering and hepatic sequestration of chylomicron remnants in LDLR-deficient mice
}

\author{
Kenneth C.-W. Yu, ${ }^{1,2}$ Wei Chen, ${ }^{2}$ and Allen D. Cooper ${ }^{1,2}$ \\ ${ }^{1}$ Department of Medicine, Division of Gastroenterology, Stanford University School of Medicine, Stanford, California, USA \\ ${ }^{2}$ Department of Cardiovascular Biology, Research Institute, Palo Alto Medical Foundation, Palo Alto, California, USA
}

Address correspondence to: Allen D. Cooper, Research Institute, Palo Alto Medical Foundation, Palo Alto, California 94301, USA. Phone: (650) 326-4821; Fax: (650) 329-9114; E-mail: adc@leland.stanford.edu.

Received for publication November 10, 2000, and accepted in revised form April 19, 2001.

\begin{abstract}
It has been proposed that in the liver, chylomicron remnants (lipoproteins carrying dietary lipid) may be sequestered before being internalized by hepatocytes. To study this, chylomicron remnants labeled with a fluorescent dye were perfused into isolated livers of LDL receptor-deficient (LDLR-deficient) mice $\left(\mathrm{Ldlr}^{-/}\right)$and examined by confocal microscopy. In contrast to livers from normal mice, there was clustering of the chylomicron remnants on the cell surface in the space of Disse. These remnant clusters colocalized with clusters of LDLR-related protein (LRP) and could be eliminated by low concentrations of receptor-associated protein, an inhibitor of LRP. When competed with ligands of heparan sulfate proteoglycans (HSPGs), the remnant clusters still appeared but were fewer in number, although syndecans (membrane HSPGs) colocalized with the remnant clusters. This suggests that the clustering of remnants is not dependent on syndecans but that the syndecans may modify the binding of remnants. These results establish that sequestration is a novel process, the clustering of remnants in the space of Disse. The clustering involves remnants binding to the LRP, and this may be stabilized by binding with syndecans, eventually followed by endocytosis.
\end{abstract}

J. Clin. Invest. 107:1387-1394 (2001).

\section{Introduction}

Dietary lipids, including triglycerides, cholesterol, and fat-soluble vitamins, are metabolized by a two-step process. After being incorporated into large lipoproteins called chylomicrons, they pass from the lymphatics to the general circulation, where the triglycerides are selectively removed, primarily by muscle and adipose tissue (1). The residual particle containing virtually all dietary cholesterol and fat-soluble vitamins is called a chylomicron remnant and is rapidly removed by the liver (1). Given that humans are in the postprandial state for more than half of the day, defective hepatic removal of chylomicron remnants can lead to their presence in the plasma for a prolonged period. This condition has been associated with the accelerated development of atherosclerosis in patients with normal fasting lipids (2), perhaps because remnants are themselves atherogenic particles. In the liver, the hepatocytes are separated from the endothelial lining by an extracellular space called the space of Disse (3). Presently, two pathways in the liver, the LDL receptor (LDLR) and the LDLR-related protein (LRP) have been identified to be important for the endocytosis of chylomicron remnants. Several aspects of this system, however, are not completely understood.

The removal of remnant lipoproteins by the LDLR and the LRP requires an interaction with apoE, the ligand on the lipoprotein particle surface (4). In the presence of the LDLR, hepatic removal and endocytosis of chylomicron remnants are rapid; however, in LDLRnull mice $\left(L d l r^{--}\right)$, in which the LRP is the dominant receptor, Herz et al. have shown that hepatic removal of chylomicron remnants was unaffected but endocytosis was delayed (5). The LRP may be a more complicated receptor than the LDLR. Factors such as heparan sulfate proteoglycans (HSPGs) and hepatic-localized apoE may influence the LRP.

It has been proposed that initially there is sequestration of remnant lipoproteins via cell-surface HSPGs in the liver (6). This is possible given that basic fibroblast growth factor (bFGF) binds to HSPGs as well as its specific receptor (7). It has been proposed that the sequestration may also involve hepatic-localized apoE, which may increase the binding of remnants to the LRP (6).

The aim of the study was to determine how sequestration of chylomicron remnants occurs and whether it is mediated by the LDLR, the LRP, or HSPGs. To do this, chylomicron remnants were labeled with a fluorescent dye and were perfused into the isolated livers of mice that were then examined by confocal microscopy. The $\mathrm{Ldlr}^{-/-}$mouse was used as the model for LRP-mediated removal of chylomicron remnants. It was found that the sequestration step involves a unique clustering of the chylomicron remnant on the cell surface mediated primarily by the LRP. The chylomicron remnantLRP clusters may be strengthened by further binding with syndecans (a family of HSPGs). 


\section{Methods}

Lipid and protein determination. Cholesterol and triglycerides were assayed using Sigma Chemical Co. kits (St. Louis, Missouri, USA). Protein was assayed using the Pierce Chemical Co. micro-BCA kit (Rockville, Illinois, USA).

Animals. Sprague-Dawley rats were from Simonsen Laboratories (Gilroy, California, USA). Ldlr-/- mice (C57BL/6J or B6.129) and wild-type (WT) mice (C57BL/6J or B6,129) were from The Jackson Laboratory (Bar Harbor, Maine, USA).

Chylomicron remnants and $\beta$-VLDL. Chylomicron remnants were obtained from the plasma of hepatectomized rats given lymph chylomicrons intravenously (8). They were labeled with carrier-free $\mathrm{Na}^{125} \mathrm{I}$ (Amersham Life Sciences Inc., Arlington Heights, Illinois, USA) by a modified McFarlane method as reported previously (8). The lipid and protein composition of the chylomicron remnants were similar to those published previously $(9,10)$.

To validate the use of rat remnants with mouse tissue, receptor-ligand binding assays were carried out using mouse L929 fibroblasts with mouse $\beta$ VLDL. $\beta$ VLDL was isolated by density gradient ultracentrifugation from the plasma of $\mathrm{Ldlr}^{/-}$mice fed a cocoa-butter diet (Test Diet, Richmond, Indiana, USA) for 14 days and labeled with ${ }^{125} \mathrm{I}(10) .{ }^{125} \mathrm{I}$-rat remnants or ${ }^{125} \mathrm{I}$-mouse $\beta$ VLDL were competed with unlabeled rat remnants or mouse $\beta$ VLDL. There was a very high inhibition (>70\%) of cell-surface binding $\left(4^{\circ} \mathrm{C}\right)$ and cell-associated radioactivity $\left(37^{\circ} \mathrm{C}\right)$ when the lipoproteins from one species were competed with the other, and the potency as competitors was virtually identical (data not shown). This and our previous studies demonstrated the feasibility of using rat remnants $(9,10)$.

Fluorescent labeling. The chylomicron remnants were labeled with the fluorescent carbocyanine dye, 1,1'dioctadecyl-3,3,3',3'-tetramethylindodicarbocyanine perchlorate (DiD) (Molecular Probes Inc., Eugene, Oregon, USA), as described previously (11). Asialofetuin was labeled with Oregon-green (OG) using a kit from Molecular Probes Inc.

Liver perfusion. The livers of 15 -week-old mice were perfused using the single pass non-recirculating procedure previously described in studies using ${ }^{125} \mathrm{I}$-remnants (8). The perfusate solution contained rat erythrocytes (20\% hematocrit) in DMEM gassed with $20 \%$ $\mathrm{O}_{2}$. The liver was thermostatically maintained at $37^{\circ} \mathrm{C}$. After a 5-minute perfusion to remove blood, the perfusate solution containing DiD remnants and/or other materials was perfused into the liver via the portal vein for 20 minutes $(0.5 \mathrm{ml} / \mathrm{min})$ and then for 5 minutes with $0.9 \% \mathrm{NaCl}$. Receptor-associated protein (RAP) was perfused as described previously (10). The livers were fixed in PBS with 4\% paraformaldehyde (15 minutes) and in PBS $+20 \%$ sucrose (16 hours). Tissue blocks were embedded in OCT, and sections $(8 \mu \mathrm{m})$ were cut and placed onto glass slides.

Immunostaining and confocal microscopy. The sections were incubated in PBS $+0.1 \%$ Triton X100 (5 minutes), in PBS $+3 \%$ BSA (30 minutes), and then with antibodies. For staining endothelial cells (ECs), an anti-vWF antibody (Sigma Chemical Co.) was used followed by OGconjugated anti-IgG (Molecular Probes Inc.). For staining LRP, a rabbit antibody prepared to the LRP amino acid sequence from 1961 to 2120 was used. The antigen was extracted from bacteria transfected with a vector encoding this sequence. The peptide was purified by SDS-PAGE. The antibody is monospecific and recognizes only the extracellular domain of the LRP. It was tested for monospecificity by blotting against membrane from several tissues including liver (data not shown). For staining syndecans, three antibodies (a gift from M. Bernfield, Harvard University, Boston, Massachusetts, USA) were used. They were a rat monoclonal IgG (no. 281.2) against mouse syndecan-1 (sy1) (12), a rat monoclonal IgM (F90) against mouse syndecan-2 (sy2), and a rat monoclonal IgG/IgM (KY8.2) against mouse syndecan-4 (sy4). OG-labeled anti-IgG or antiIgM (Molecular Probes Inc.) was used as appropriate.

Digital images of the stained sections were obtained using a Molecular Dynamics Multiprobe confocal laser microscope (Sunnyvale, California, USA). DiD was excited at 644-nm. OG was excited at 488-nm. A greater than 660-nm and a 500- to 560-nm filter were used to collect DiD emission (channel 1: red) and OG emission (channel 2: green), respectively. To determine colocalized pixels, a graph of red versus green pixels was generated using the Molecular Dynamic's ImageSpace software. NIH Image software (version 1.62; NIH, Bethesda, Maryland, USA) was used to calculate the percent of colocalization (expressed as relative to number of total pixels).

The number of clusters of chylomicron remnants was quantified from the digital images. A cluster is defined as a single continuous "streak" or "clump" of red fluorescence located on the cell surface. Sections were selected using a random number protocol.

Data analysis. Where appropriate, the data are expressed as the mean \pm SEM ( $n=$ number of animals). Student's $t$ test was used to determine significance between groups. A $P$ value of less than 0.05 was deemed significant.

\section{Results}

Uptake of asialofetuin and chylomicron remnants by the perfused liver. Asialofetuin is endocytosed by a receptormediated pathway (13). A solution containing equal amounts ( $4 \mu \mathrm{g}$ protein per milliliter) of DiD remnants and OG-labeled asialofetuin was perfused into WT mouse livers for 20 minutes. There was uptake of DiD remnants (Figure 1a, red) and OG-asialofetuin (Figure 1a, green) predominantly in hepatocytes (Figure 1, a-c). A plot of OG against DiD pixels (Figure 1d) showed that colocalization (yellow) of the two particles was $68.78 \pm 5.06 \%(n=5)$, and this indicates that remnants are taken up by a similar receptor-mediated endocytotic pathway(s). ECs were stained with an anti-vWF antibody (green) (Figure 1e). The hepatocytes are clearly identified by the red fluorescence from the endocyto- 

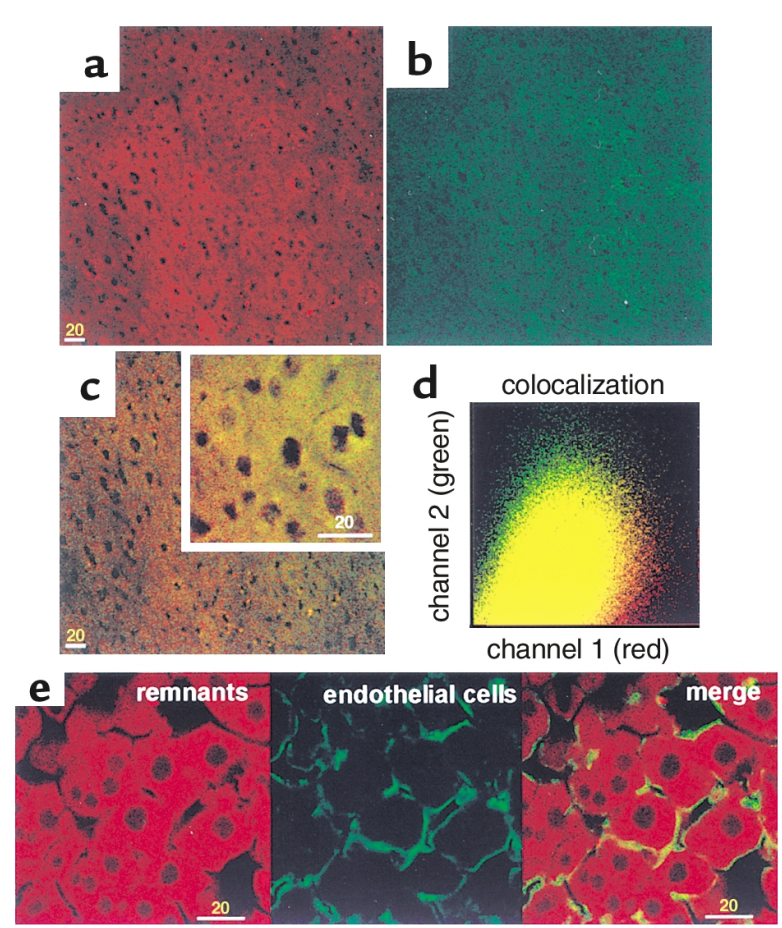

\section{Figure 1}

Uptake of chylomicron remnants and asialofetuin in wild-type (WT) livers. Livers were perfused with $4 \mu \mathrm{g} / \mathrm{ml}$ of both DiD remnants and OG-asialofetuin; tissue sections were examined by confocal microscopy, and digital images were obtained as described in Methods. (a) A representative image of the uptake of DiD remnants (red) and (b) OG-asialofetuin (green) in a liver section. (c) The merged image of $\mathbf{a}$ and $\mathbf{b}$. The inset shows higher magnification. (d) The graph of colocalized pixels in c. In separate experiments in which only DiD remnants were perfused, ECs were stained with OG-labeled antivWF antibody. (e, left) DiD remnants; (e, center) ECs; (e, right) a merged image of left and center images. Bars $=20 \mu \mathrm{m}$.

sis of DiD remnants. The space of Disse is not generally visible at this level of magnification, but it is defined as that between the EC lining the sinusoids and the hepatocytes. The percent of colocalization of the two labels was $16.15 \pm 0.32 \%(n=5)$. This indicates that there is distinct staining of ECs with little problem of bleed-through between the two channels. There was a nearly linear increase in the DiD-remnant fluorescence intensity with increasing perfusion time $(5,10,15$, and 20 minutes) (Figure 2). In pulse chase experiments, the remnants moved from the cell surface to the perinuclear regions over this period (data not shown). These data demonstrate that in the perfused livers of normal mice at physiological concentrations, remnants are efficiently internalized by receptor-mediated endocytosis. The concentration of chylomicron remnants used for subsequent studies was $4 \mu \mathrm{g} / \mathrm{ml}$. In our previous studies using $4 \mu \mathrm{g} / \mathrm{ml}$, the removal of ${ }^{125}$ I-remnants by $L d l r^{-1}$ livers was efficient but modestly less than in WT livers (8). In the absence of the LDLR, the LRP is presumed to be the dominant receptor. This suggests that $4 \mu \mathrm{g} / \mathrm{ml}$ is above the $\mathrm{K}_{d}$ for the LRP but that it is not saturated with remnants.
Clustering of chylomicron remnants in Ldlr-/- livers. In $\mathrm{Ldlr}^{-1-}$ mouse livers, although the total remnant removal was decreased by $20 \%$ (8), the endocytosis of DiD remnants was decreased to a greater degree. After the 20-minute perfusion, the total fluorescence intensity of remnants in $\mathrm{Ldlr}^{-/-}$livers was still visible but markedly less than in WT livers (Figure 3a). The pattern of remnant distribution was strikingly different. In WT livers, the distribution of remnants was diffuse but homogenous throughout the cytosol (Figure $3 \mathrm{~b}$ ). The occasional large punctuate clumps in the cytosol probably represent localization in the perinuclear region where fusion of endosomes with lysosomes occur. In $\mathrm{Ldlr}^{-1}$ livers, there was less uptake into the hepatocytes; the remnants appeared as smaller punctuate clumps in the cytosol (Figure 3c). Surprisingly, distinct clusters of remnants appeared randomly on the cell surface. These clusters of remnants looked like aggregates that accumulated on the cell surface in close proximity with ECs (Figure 3c). Some were large whereas others were smaller. In contrast, there were substantially fewer clusters of chylomicron remnants in WT livers. The number of clusters in the WT livers and in $\mathrm{Ldlr}^{-/-}$livers was $2.93 \pm 0.38(n=5)$ and $17.87 \pm 0.41(n=5)$ per $45,000 \mu \mathrm{m}^{2}$, respectively. Increasing the remnant concentration perfused through the WT livers to $8 \mu \mathrm{g} / \mathrm{ml}$ did not increase the number of clusters, although it did increase the amount of remnants taken up by the livers. The number of clusters after perfusion of 4 $\mu \mathrm{g} / \mathrm{ml}$ or $8 \mu \mathrm{g} / \mathrm{ml}$ of remnants in a separate set of experiments was $3.0 \pm 0.57(n=3)$ and $4.0 \pm 1.15$ $(n=3)$ per $45,000 \mu \mathrm{m}^{2}$, respectively whereas DiD-remnant fluorescence intensity was $16.33 \pm 0.6 \times 10^{6}$ units $(n=3)$ and $23.64 \pm 0.8 \times 10^{6}$ arbitrary fluorescence units $(n=3)$, respectively. In $L d l r^{-/}$livers, reducing the remnant concentration from 4 to $2 \mu \mathrm{g} / \mathrm{ml}$ reduced the number of clusters but did not change the proportion of the clusters to the amount taken

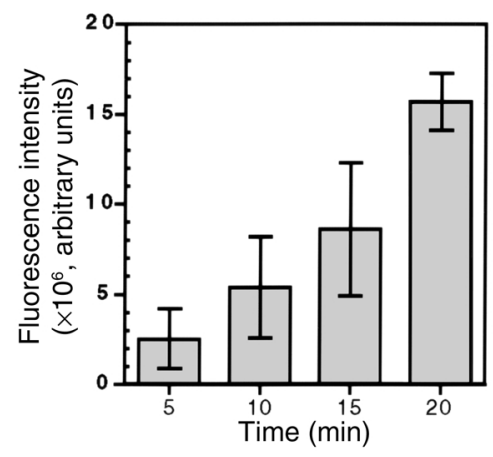

\section{Figure 2}

Chylomicron remnant uptake with time in WT livers. Livers were perfused with $4 \mu \mathrm{g} / \mathrm{ml}$ DiD remnants for the indicated times. Digital images of liver sections were obtained by confocal microscopy, and the total sum of the pixels (fluorescence) in each image was calculated. The data are expressed as fluorescence intensity (arbitrary units) and are the mean $\pm \operatorname{SEM}(n=5)$. 

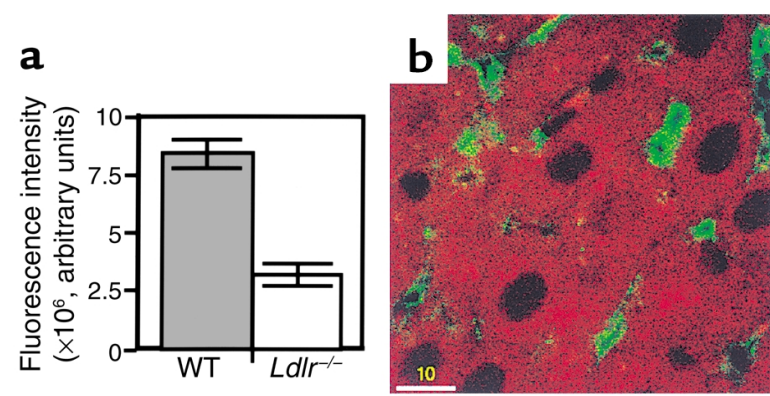

Figure 3

Chylomicron remnant uptake in WT and $L d l r^{-/}$livers. Livers were perfused with $4 \mu \mathrm{g} / \mathrm{ml}$ DiD remnants (for 20 minutes), and cut sections were examined as described in Figure 1. (a) Fluorescence intensity of DiD remnants in WT and $L d l r^{\prime-}$ liver sections was determined as described in Figure 2. Data are the mean $\pm \operatorname{SEM}(n=10)$. (b) Uptake of DiD remnants (red) in a WT liver section. (c) Clustering and uptake of DiD remnants in an $L d l r^{-1-}$ liver section. ECs stained with OG-labeled anti-vWF antibody are in green. Bars $=10 \mu \mathrm{m}$.

up. At $4 \mu \mathrm{g} / \mathrm{ml}$, the number of clusters was $26.07 \pm 0.9 / 45,000 \mu \mathrm{m}^{2}(n=3)$ and DiD remnant taken up was $9.5 \pm 0.37 \times 10^{6}$ arbitrary fluorescence units. At $2 \mu \mathrm{g} / \mathrm{ml}$, the number of clusters was significantly reduced to $13.07 \pm 1.5 / 45,000 \mu \mathrm{m}^{2}(n=3)$ $(P=0.02)$, whereas DiD remnant taken up was also significantly reduced to $5.15 \pm 0.84 \times 10^{6}$ arbitrary fluorescence units $(n=3)(P=0.03)$. At $8 \mu \mathrm{g} / \mathrm{ml}$ in the $L d l r^{--}$livers, the number of clusters and DiD remnants taken up did not increase significantly, consistent with the postulate that the system was nearing saturation. The number of clusters was $27.63 \pm 0.37$ / $45,000 \mu \mathrm{m}^{2}(n=3)$, and DiD remnant taken up was $10.9 \pm 0.52 \times 10^{6}$ arbitrary fluorescence units.

Clustering of chylomicron remnants in the extracellular space. To ascertain whether remnant clusters were intraor extracellular, $0.05 \%$ trypan blue was perfused into the livers after an initial 20-minute perfusion with DiD remnants. Trypan blue quenches fluorescence and does not penetrate intact cell membranes $(14,15)$. There was little change seen in the distribution of fluorescence in

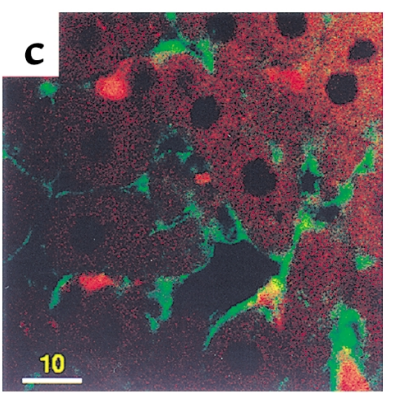

WT livers in the presence or absence of trypan blue (Figure 4 , $a$ and $b$ ). In contrast, the number of remnant clusters in the $\mathrm{Ldlr}^{-/}$livers after trypan blue perfusion was reduced by $95 \%$ (Figure 4, d and e). The number of clusters in livers with or without trypan blue was $21.16 \pm$ $1.93 / 45,000 \mu \mathrm{m}^{2}(n=5)$ and $1.04 \pm 0.27 / 45000 \mu \mathrm{m}^{2}(n=5)$, respectively. This demonstrated that the clustering of remnants takes place on the cell surface in the extracellular space (space of Disse).

Clustering of chylomicron remnants is mediated by the LRP. The RAP, a ligand of all receptor sites on the LRP (16), was used to compete with DiD remnants in $\mathrm{Ldlr}^{-/}$livers. A concentration of RAP of 4 $\mu \mathrm{g} / \mathrm{ml}$ was chosen because it blocks binding to the LRP but does not affect binding to other LDLR family members (8). RAP almost completely eliminated the clustering of remnants. There was still detectable uptake of remnants by the hepatocytes (Figure 4, $\mathrm{c}$ and $\mathrm{f}$ ). This strongly indicates that the clustering is dependent on the LRP. The continued uptake of chylomicron remnants, despite the presence of RAP, suggests that either the LRP was not completely inhibited or there are specific and/or nonspecific non-LDLR/non-LRP pathways that can lead to the uptake of small amounts of remnants.

The cellular location of LRP was examined. Before perfusion of DiD remnants, LRP, detected by immunofluorescence staining using a monospecific antibody to an extracellular domain of the LRP, was distributed evenly as thin strings on the cell surface with little in the cytosol (Figure 5a). Interestingly, there was a higher staining of the LRP at the apices of the cells. After

\section{Figure 4}

Effect of trypan blue and RAP on chylomicron remnant uptake in $L d l r^{-}-$livers. (a and b) WT and (d and e) $L d l r^{-}$- livers were perfused with $4 \mu \mathrm{g} / \mathrm{ml}$ DiD remnants (red) and further perfused with (b and $\mathbf{e}$ ) trypan blue. In separate experiments, the perfusate contained RAP $(4 \mu \mathrm{g} / \mathrm{ml})$ and was not followed by trypan blue perfusion in (c) WT and (f) Ldl $r^{-1}$ - livers. ECs are in green. Bars $=10 \mu \mathrm{m}$.
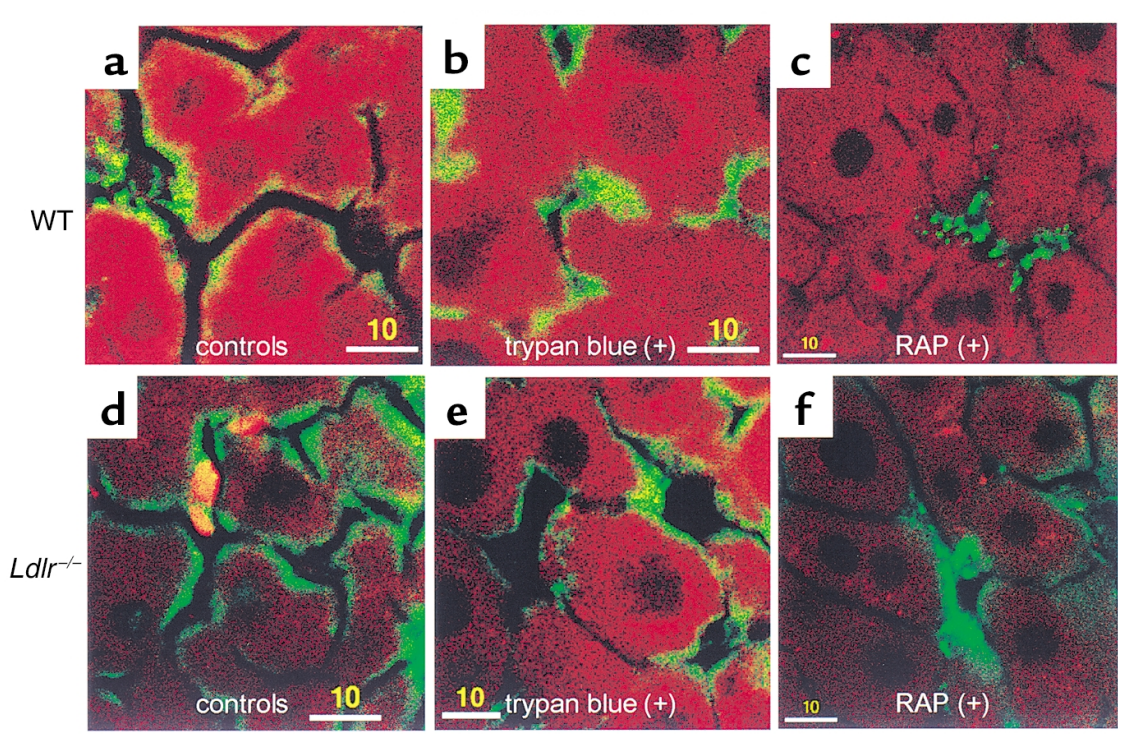


\section{Figure 5}

Effect of LRP competitors on LRP distribution and chylomicron remnant uptake in $L d l r^{--}$livers. Experiments were conducted as described in Figure 1. LRP was stained with anti-LRP antibody (green). The perfusate contained only media or DiD remnants (red) and unlabeled competitors. (a) $L d l r^{-/}$livers perfused with lipoprotein-free media. (b-d) $L d l r^{-/}$livers perfused with $4 \mu \mathrm{g} / \mathrm{ml}$ DiD remnants. (e-g) $L d l r^{-}-$livers perfused with $4 \mu \mathrm{g} / \mathrm{ml}$ DiD remnants and $8 \mu \mathrm{g} / \mathrm{ml}$ $\alpha_{2}$-macroglobulin $\left(\alpha_{2} M\right)$. (h-j) $L d l r^{-1}$-livers perfused with $4 \mu \mathrm{g} / \mathrm{ml}$ DiD remnants and $8 \mu \mathrm{g} / \mathrm{ml}$ RAP. (b, e, and $\mathbf{h})$ DiD remnants; (a, c, f, and $\mathbf{i}) L R P ;(\mathbf{d}, \mathbf{g}$, and j) merged images. Bars $=10 \mu \mathrm{m}$.

perfusion of DiD remnants, distinct aggregates or clusters of LRP formed on the cell surface (Figure 5c). The majority of these clusters colocalized with the remnant clusters, and there was increased staining of LRP in the cytosol (Figure 5, b and d). Thus, chylomicron remnants causes clustering of LRP coincident with clusters of remnants.

The effect of $\alpha_{2}$-macroglobulin $\left(\alpha_{2} \mathrm{M}\right)$, another LRP ligand, on remnant removal was also studied. The $\alpha_{2} \mathrm{M}$ was activated with trypsin as described previously (10) and then perfused with DiD remnants into $\mathrm{Ldlr}^{-/-}$livers. Clusters of remnants and LRP were still formed (Figure 5, e-g).

This suggested that $\alpha_{2} \mathrm{M}$-trypsin may bind to a site on the LRP different from that to which remnants bind. This is consistent with the small effect of $\alpha_{2} \mathrm{M}$ seen in previous studies (8). Again, RAP was used and found to inhibit clustering of LRP (Figure 5, h-j). This was expected because RAP and remnants share the same binding site on LRP (17).

Role of HSPGs. There are three syndecans (a family of HSPGs) expressed by the liver. They are sy1, sy2, and sy 4 (7). In control experiments in which $\mathrm{Ldlr}^{-/}$livers were perfused with lipoprotein-free media, the syndecans were distributed evenly on the plasma membrane with no aggregation (data not shown). In contrast, after perfusion with DiD remnants, clusters of remnants formed (Figure 6a) and discrete aggregates of syndecans (sy1) were stained intensely (Figure 6b). A similar result was also obtained with sy 2 and sy4 (data not shown). The
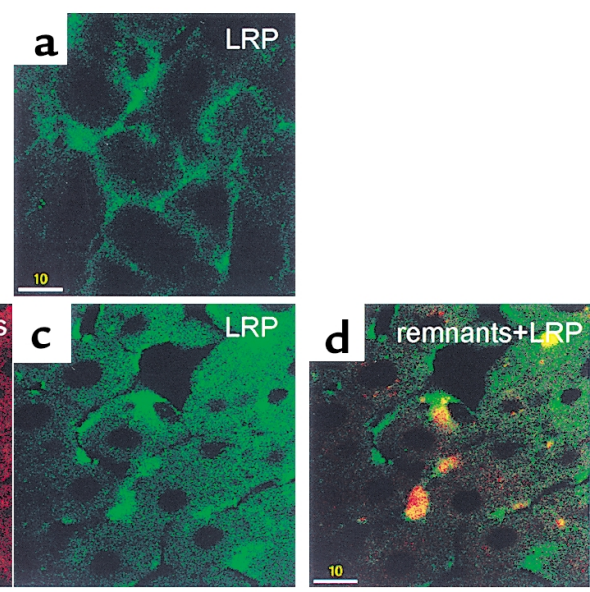

ipoprotein-free media
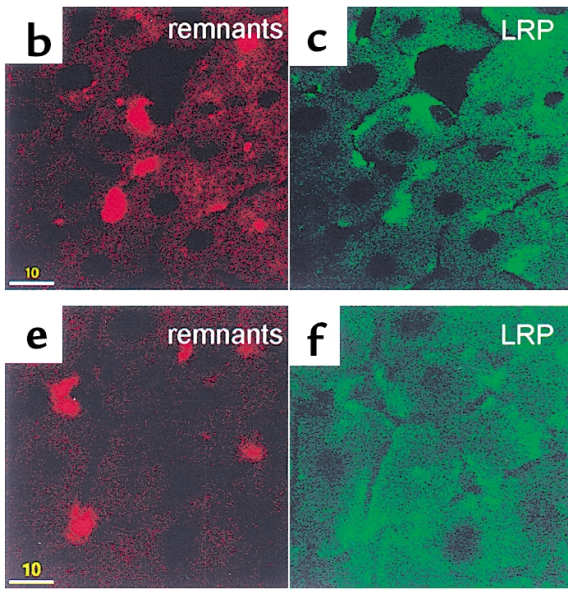

$R P$
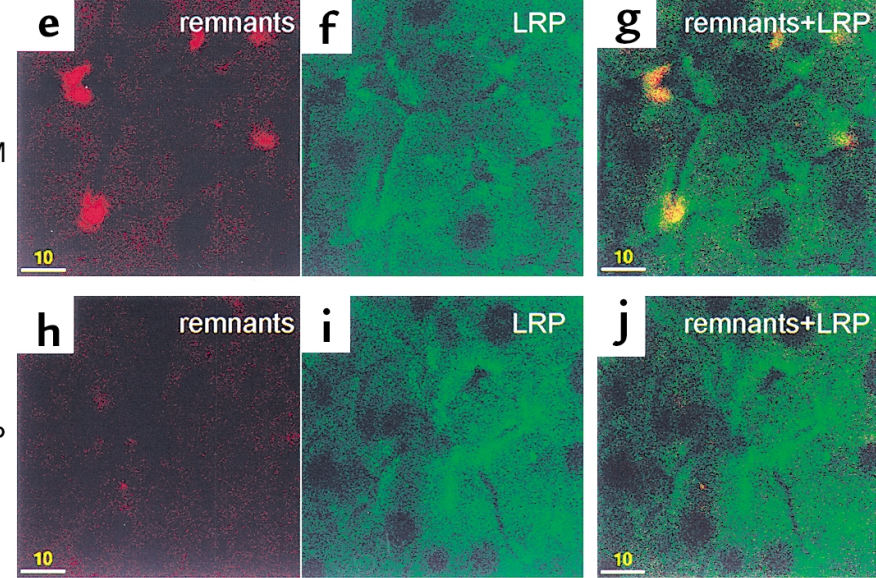

clusters of sy1 colocalized with the clusters of chylomicron remnants (Figure 6c). Colocalization of sy 2 and sy 4 clusters with remnant clusters were also seen (data not shown), suggesting that there is binding of syndecans with the remnant-LRP clusters.

Two ligands of HSPGs, Na heparin and bFGF (7), were added to the perfusate to compete with DiD remnants. A low heparin concentration $(0.5 \mu \mathrm{g} / \mathrm{ml})$ did not affect the clustering of remnants; nor did bFGF $(0.5$ $\mu \mathrm{g} / \mathrm{ml}$ ) (data not shown). Higher concentrations of these HSPG ligands $(6 \mu \mathrm{g} / \mathrm{ml})$ reduced but did not completely eliminate the clustering (Figure 7, a-c). The number of remnants clusters in the livers without heparin was $18.93 \pm 0.37 / 45,000 \mu \mathrm{m}^{2}(n=3)$ and that in livers with heparin $(6 \mu \mathrm{g} / \mathrm{ml})$ was $13.47 \pm 0.48 /$ $45,000 \mu \mathrm{m}^{2}(n=3)$. The number of remnant clusters in the livers without bFGF was $22.88 \pm 0.6 / 45,000 \mu \mathrm{m}^{2}$

\section{Figure 6}

Syndecans colocalize with clusters of chylomicron remnants. $L d l r^{-/}$livers were perfused with DiD remnants (20 minutes), and cut sections were immunostained with OG-labeled anti-syndecan-1. There was (a) clustering of DiD remnants (red) that was colocalized with (b) syndecan-1 (green). (c) The merged image of $\mathbf{a}$ and $\mathbf{b}$ with intense staining of syndecan-1 colocalized with remnant clusters. Bars $=10 \mu \mathrm{m}$.

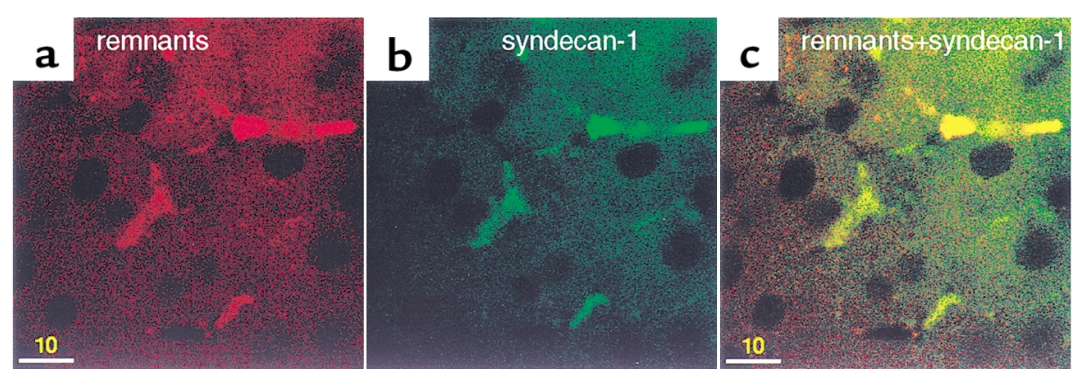




\section{Figure 7}

Effect of $\mathrm{Na}$ heparin and bFGF on chylomicron remnant uptake in $L d / r^{-1-}$ livers. $L d / r^{-/}$livers were perfused with DiD remnants (red) (a) without or (b) with $6 \mu \mathrm{g} / \mathrm{ml} \mathrm{Na}$ heparin or (c) $6 \mu \mathrm{g} / \mathrm{ml} \mathrm{bFGF}$, and cut sections were examined as described in Figure 1. ECs are in green. Bars $=10 \mu \mathrm{m}$. In separate experiments, 125I-remnants were perfused into livers of (d) WT and (e) $L d l r^{-1}$ - livers without or with $\mathrm{Na}$ heparin or bFGF. The amount removed per pass (\% of total perfused per pass) is plotted as a function of time. ( $\mathbf{f}$ ) Total liver uptake of 125 I-remnants was also determined and is expressed as micrograms of protein of ${ }^{125} \mathrm{I}$-remnants taken up per gram of liver. Data for $\mathbf{d}-\mathbf{f}$ are the mean $\pm \operatorname{SEM}(n=3)$.

$(n=3)$ and that in livers with bFGF $(6 \mu \mathrm{g} / \mathrm{ml})$ was $18.8 \pm 0.49 / 45,000 \mu \mathrm{m}^{2}(n=3)$.

The total DiD-remnant fluorescence intensity of liver sections is not a precise measure of total liver uptake of remnants. To obtain a more direct quantification of the effects of HSPG inhibitors on total liver uptake (amount of remnants in the livers), ${ }^{125} \mathrm{I}$-remnants were perfused with $6 \mu \mathrm{g} / \mathrm{ml}$ of $\mathrm{Na}$ heparin or bFGF. The percent of ${ }^{125}$ I-remnants removed from the perfusate per minute (pass) and the total liver uptake of ${ }^{125}$ I-remnants were determined as described previously (8). In WT livers, the ${ }^{125}$ I-remnants removed per pass was $50-60 \%$ at 20 minutes (Figure 7d). This was not affected by heparin or bFGF (Figure 7d). Similarly, the amount of ${ }^{125}$ I-remnants in the livers was not changed by heparin or bFGF (Figure 7f, filled bars). In $\mathrm{Ldlr}^{-/-}$mice, the ${ }^{125} \mathrm{I}$-remnants removed per pass was $30-40 \%$ (Figure 7e). The presence of heparin or bFGF caused a decrease of 10-15\% (Figure $7 \mathrm{e})$; however, this was not significant $(P=0.13$ for heparin, $P=0.09$ for $b F G F$ ). Although there was a small decrease in the amount of remnants in the liver in the presence of heparin or bFGF, it was insignificant (Figure 7f, open bars). Thus, although HSPGs are not necessary for the clustering of remnants, their absence may modestly reduce remnant uptake.

\section{Discussion}

The present study establishes that in the absence of the LDLR, a unique processing of chylomicron remnants occurs in the liver. At physiologic concentrations of remnants, there is only a modest reduction in the uptake of remnants in livers of $\mathrm{Ldlr}^{-/-}$mice compared to normal livers. The remnant concentration that exists postprandially in vivo is not known precisely but from the masses of apoB48 and triglycerides, it can be calculated that $2-20 \mu \mathrm{g} / \mathrm{ml}$ of remnant protein is in the physiologic range. In $\mathrm{Ldlr}^{\prime-}$ livers, however, fewer fluorescent remnants appeared in the liver cells. These probably were endocytosed by the LRP. A substantial portion of the remnants, however, were localized as controls
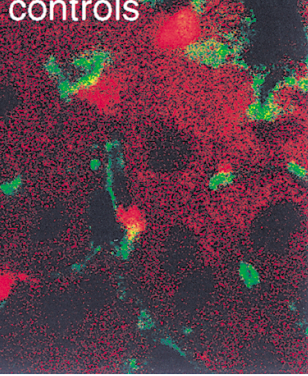

\section{heparin}
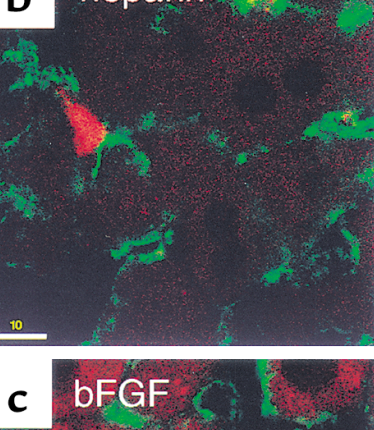

c $\mathrm{bFGF}$

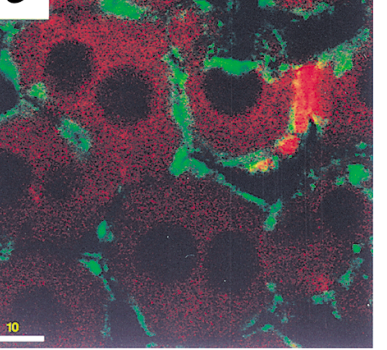

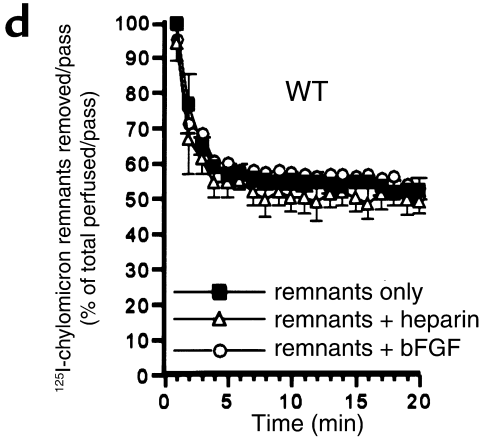

e

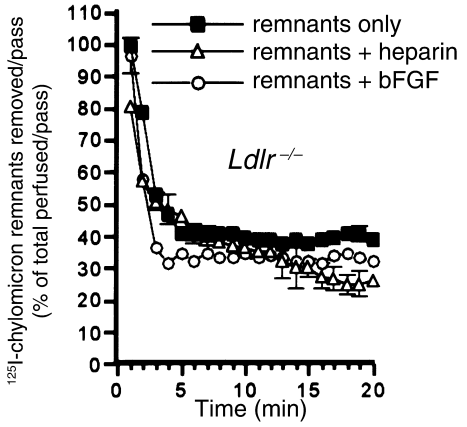

f

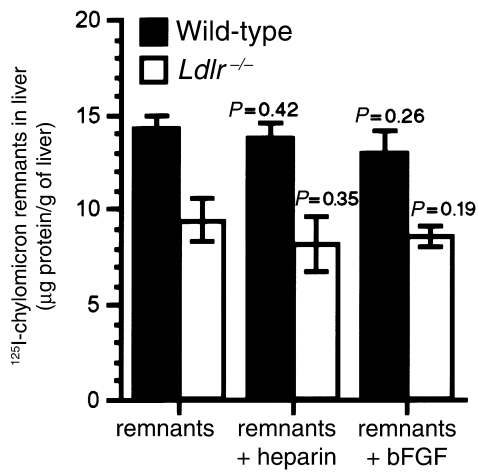

clusters on the cell surface. These clusters may explain the findings of Herz et al. (5), that endocytosis by the LRP is relatively slow in $\mathrm{Ldll}^{-/}$- livers. Accordingly, the determinants of clustering of remnants was investigated in $\mathrm{Ldll}^{-/}$- livers.

The clusters of remnants had only a partial overlap with either the hepatocytes or the ECs lining the hepatic sinusoids. The experiments using trypan blue, a fluorescence quencher that does not enter cells, established that the clusters were in the space of Disse. It has been proposed that remnants are initially removed by a process called sequestration or secretion-capture (6). The clustering of the remnants is probably the anatomic basis for sequestration. To identify the molecules required for the clustering, inhibitors of LRP binding were studied. RAP almost completely inhibited the formation of clusters, but it did not completely block remnant uptake. Previously, our laboratory showed that there was some remnant uptake in the $\mathrm{Ldl}^{-/}$- livers in the presence of RAP; however, this is relatively small (8). This may be due to unblocked LRP because of the low RAP concentration, or to the existence of other receptors. This is quantitatively a small amount and is not 
due to the exchange of the dye, as there was virtually no dye in cultured fibroblasts when they were incubated with DiD remnants with RAP and anti-LDLR antibodies (data not shown). Thus, we conclude that the LRP is required for the clustering of remnants.

The role of HSPGs was investigated by using bFGF as a competitor because it binds to HSPGs (7). At a concentration that should saturate all of the HSPG binding sites, bFGF did not affect remnant removal or clustering. Given that bFGF might not bind to the same site as apoE, heparin was used (18). Heparin will displace apoE from HSPGs, thus the site of binding for apoE and heparin on HSPG should be identical. The caveat to this approach is that heparin has numerous effects on lipoprotein metabolism that might decrease remnant removal without disturbing the interaction of lipoproteins with HSPGs (19). Despite this possibility, heparin at either a low concentration that generally inhibits binding or at a high concentration that should interfere with all heparin-sensitive interactions did not affect the clustering of remnants. The amount of heparin used was such that it would have eluted apoE from all of the sites in the liver if the liver was a heparinSepharose column. The shape of the ${ }^{125}$ I-remnant removal curve in the presence of heparin was not significantly different from controls. Heparin did reduce, but not significantly, the total amount of ${ }^{125}$ I-remnants taken up by the liver compared with controls. Thus, heparin might be affecting the affinity of the receptor for the remnants. Heparitinase, which removes HSPGs, has been used in vitro and in vivo (6). In those studies, removal of HSPGs markedly decreased the cellular uptake of apoE-enriched $\beta$ VLDL. Perhaps $\beta V L D L$ removal by the liver requires HSPGs. Heparitinase was not used in this study because it was observed that it disrupted ECs and distorted the mouse liver architecture after perfusion (data not shown). Our present data suggest that HSPGs are not critical for the clustering and are not necessary for remnant removal by the LRP, although HSPGs may affect the affinity of the process. This also contradicts the hypothesis that HSPGs are endocytic receptors for lipoproteins (20).

The role of hepatic-localized apoE in the clustering of remnants was only examined briefly in the present study. In our previous studies using ${ }^{125}$ I-remnants, $a_{p o e^{-/-}}$mouse livers removed remnants normally as long as the remnants contained an adequate amount of apoE (8). In the absence of apoE, there is no removal of the remnants by apoe $e^{-/-}$livers, but apoE-deficient remnants could be removed by livers that secrete apoE. The remnants used in our present study all contained apoE; the apoE is derived from the periphery and thus is primarily of hepatic origin. In experiments not shown, we found that there was clustering of remnants after perfusion of livers of $\mathrm{Ldll}^{-/} / \mathrm{apoe}^{-/}$mice. The remnants contained apoE; we did not determine whether the $\mathrm{Ldll}^{-/-} / \mathrm{apoe}^{-/-}$hepatocytes took up the remnants, nor did we test the fate of apoe $e^{-/-}$remnants. Thus, although it appears that apoE of hepatic origin acquired in the periphery is required for clustering of remnants, it does not appear that hepatic-localized apoE in the liver is also required for clustering at least at a low concentration of remnants. Previously, Linton et al. (21) transplanted bone marrow from normal mice

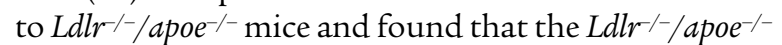
livers had abundant amounts of macrophage-derived apoE but that plasma removal of remnants was not improved. They postulated that hepatic endocytosis of remnants by the LRP requires hepatic apoE. Macrophage apoE, they proposed, is defective in binding to the LRP. It may be, however, that overabundance of hepatic-localized apoE, whether it be of macrophage or hepatic origin, may interfere with either binding or endocytosis by the LRP or both. If the overabundance of hepatic-localized apoE does interfere with binding, it is likely that clustering of remnants is inhibited. Veniant et al. (22) have shown that apoB48-containing lipoproteins are taken up by the LRP more efficiently than are apoB100-containing lipoproteins. They suggested that apoB100 may limit the amount of apoE that can be acquired by the lipoproteins from the periphery, thus lipoproteins may be dependent on "supplemental hepatic apoE" for uptake by the LRP. The fact that chylomicron remnants, which contain only apoB48, are able to acquire sufficient apoE during their formation in the periphery to allow uptake by the LDLR and/or LRP may explain why they are more effectively removed than VLDL remnants and perhaps even $\beta V L D L$ that contains considerably more apoB100 than apoB48. Thus, the two studies are in concert. Whether there is clustering of apoB100-containing lipoproteins by the LRP in the liver is an interesting question. The clustering may depend on the amount of particle apoE. It is established here that there is clustering of apoB48containing lipoproteins; however, apoB100-containing lipoproteins may not cluster, there may be very little clustering or clustering of apoB100 remnants may require hepatic-localized apoE for clustering. This should be the subject of future studies.

Staining of LRP and syndecans provided additional insights. Normally, the LRP and syndecans are uniformly distributed on the cell surface. After perfusion with remnants, both of these molecules redistributed and became clustered in the same location as the remnant clusters. This suggests that remnants are retained on the cell surface by a complex of syndecans and LRP. The data on ${ }^{125} \mathrm{I}$-remnants and HSPG ligands suggest that HSPGs are not critical for rapid removal by the liver; this further supports the hypothesis that the role of syndecans is to enhance the affinity of the LRP for remnants. This is similar to the role HSPGs play with other ligands such as bFGF and EGF (7). The enhancement of affinity may explain why "supplemental hepatic apoE" is not needed for binding to the LRP when a high level of HSPGs and particle apoE is present as it is with chylomicron remnants in the liver.

The role of hepatic lipase was not investigated. Some laboratories have shown that hepatic lipase can act as 
an additional binding site for lipoproteins in the liver $(23,24)$. Hepatic lipase is anchored to HSPGs; however, the form of hepatic lipase in the mouse is deficient in its binding to HSPGs (24). Therefore, the mouse liver has considerably less hepatic lipase compared with the rat liver. Given that our studies used mouse livers, the role of hepatic lipase is assumed to be negligible.

Why the clusters form and their physiological significance are open to speculation. The LRP certainly mediates endocytosis of chylomicron remnants. The LRP has many binding sites, and it may be that binding to some of them may not induce endocytosis but may trigger intracellular signals that initiate clustering. Another possibility is that different isoforms of the LRP exist, so that binding to one isoform triggers clustering, whereas another triggers endocytosis. The simplest explanation may be that there is extensive bridging between the LRP and remnants so that many receptors and many particles form a network that is too large to be rapidly endocytosed.

Once the clusters are formed, the fate of the remnants is of considerable interest. The clusters may serve as a reservoir to keep the remnant concentration in the circulation low while protecting the hepatocytes from the sudden infusion of triglycerides, cholesterol, and lipid-soluble toxins. The remnants may then be endocytosed by the LDLR, whose numbers will increase as intracellular sterol content falls, or by a subset of LRP that carry out an endocytic function, or most likely by both. If no sequestration of remnants occurred, lipoproteins containing dietary lipid could be in prolonged contact with the arteries and this could have pathological implications.

In summary, the model of sequestration we propose involves the binding and clustering of chylomicron remnants by the LRP as the initial step in nonLDLR-mediated remnant removal. In the normal metabolism of chylomicron remnants after a meal, the LDLR may become saturated and the remnants bound to the LRP become clustered. The clusters are retained on the cell surface for an extended period before endocytosis. HSPGs may be required for stabilizing the remnant-LRP clusters. This postulate conflicts with models that propose the binding to HSPGs as an initial step for remnant binding. The present studies carried out at a cellular level in an intact liver allow this distinction and are consistent with much, if not all, of our previous work that led to the present hypothesis $(8,10)$.

\section{Acknowledgments}

We thank Susan Palmieri (Cell Science Imaging facility, Stanford University) for confocal microscopy training, and Merton Bernfield for his gift of antibodies. This work is supported by an American Heart Association (Western States Affiliate) postdoctoral fellowship (awarded to K.C.-
W. Yu), NIH grant (DK38318), and the Stanford University Digestive Disease Center (DK38707).

1. Cooper, A.D. 1997. Hepatic uptake of chylomicron remnants. J. Lipid Res. 38:2173-2192.

2. Karpe, F. 1999. Postprandial lipoprotein metabolism and atherosclerosis. J. Intern. Med. 246:341-355.

3. Geerts, A., et al. 1986. Immunogold localization of procollagen III, fibronectin and heparan sulfate proteoglycans on ultrathin frozen sections of the normal rat liver. Histochemistry. 84:355-362.

4. Beisiegel, U., Weber, W., Ihrke, G., Herz, J., and Stanley, K.K. 1989. The LDL-receptor-related protein, LRP, is an apolipoprotein E-binding protein. Nature. 341:162-164.

5. Herz, J., et al. 1995. Initial hepatic removal of chylomicron remnants is unaffected but endocytosis is delayed in mice lacking the low density lipoprotein receptor. Proc. Natl. Acad. Sci. USA. 92:4611-4615.

6. Mahley, R.W., and Ji, Z.S. 1999. Remnant lipoprotein metabolism: key pathways involving cell-surface heparan sulfate proteoglycans and apolipoprotein E. J. Lipid Res. 40:1-16.

7. Bernfield, M., et al. 1999. Functions of cell surface heparan sulfate proteoglycans. Annu. Rev. Biochem. 68:729-777.

8. Yu, K.C., Jiang, Y., Chen, W., and Cooper, A.D. 1999. Evaluation of the components of the chylomicron remnant removal mechanism by use of the isolated perfused mouse liver. J. Lipid Res. 40:1899-1910.

9. Fujioka, Y., Cooper, A.D., and Fong, L.G. 1998. Multiple processes are involved in the uptake of chylomicron remnants by mouse peritoneal macrophages. J. Lipid Res. 39:2339-2349.

10. Yu, K.C., Jiang, Y., Chen, W., and Cooper, A.D. 2000. Rapid initial removal of chylomicron remnants by the mouse liver does not require hepatically localized apolipoprotein E. J. Lipid Res. 41:1715-1727.

11. Pitas, R.E., Innerarity, T.L., Weinstein, J.N., and Mahley, R.W. 1981. Acetoacetylated lipoproteins used to distinguish fibroblasts from macrophages in vitro by fluorescence microscopy. Arteriosclerosis. 1:177-185.

12. Saunders, S., Jalkanen, M., O’Farrell, S., and Bernfield, M. 1989. Molecular cloning of syndecan, an integral membrane proteoglycan. J. Cell Biol. 108: $1547-1556$.

13. Wahlberg, J.M., and Spiess, M. 1997. Multiple determinants direct the orientation of signal-anchor proteins: the topogenic role of the hydrophobic signal domain. J. Cell Biol. 137:555-562.

14. Myers, J.N., Tabas, I., Jones, N.L., and Maxfield, F.R. 1993. $\beta$-VLDL is sequestered in surface-connected tubules in mouse peritoneal macrophages. J. Cell Biol. 123:1389-1402.

15. Weersink, A.J., Van Kessel, K.P., Torensma, R., Van Strijp, J.A., and Verhoef, J. 1990. Binding of rough lipopolysaccharides to human leukocytes. Inhibition by anti-LPS monoclonal antibody. J. Immunol. 145:318-324.

16. Strickland, D.K., et al. 1990. Sequence identity between the $\alpha_{2}$ macroglobulin receptor and low density lipoprotein receptor-related protein suggests that this molecule is a multifunctional receptor. J. Biol. Chem. 265:17401-17404.

17. Krieger, M., and Herz, J. 1994. Structures and functions of multiligand lipoprotein receptors: macrophage scavenger receptors and LDL receptor-related protein. Annu. Rev. Biochem. 63:601-637.

18. Aviezer, D., et al. 1994. Differential structural requirements of heparin and heparan sulfate proteoglycans that promote binding of basic fibroblast growth factor to its receptor. J. Biol. Chem. 269:114-121.

19. Zsigmond, E., Fuke, Y., Li, L., Kobatashi, K., and Chan, L. 1998. Resistance of chylomicron and VLDL remnants to post-heparin lipolysis in apoE-deficient mice: the role of apoE in lipoprotein lipase-mediated lipolysis in vivo and in vitro. J. Lipid Res. 39:1852-1861.

20. Fuki, I.V., Iozzo, R.V., and Williams, K.J. 2000. Perlecan heparan sulfate proteoglycans: a novel receptor that mediates a distinct pathway for ligand catabolism. J. Biol. Chem. 275:25742-25760.

21. Linton, M.F., Hasty, A.H., Babaev, V.R., and Fazio, S. 1998. Hepatic apoE expression is required for remnant lipoprotein clearance in the absence of the low density lipoprotein receptor. J. Clin. Invest. 101:1726-1736.

22. Veniant, M.M., et al. 1998. Lipoprotein clearance mechanisms in LDL receptor-deficient "apoB48-only" and "apoB100-only" mice. J. Clin. Invest. 102:1559-1568.

23. Choi, S.Y., Goldberg, I.J., Curtiss, L.K., and Cooper, A.D. 1998. Interaction between ApoB and hepatic lipase mediates the uptake of ApoB-containing lipoproteins. J. Biol. Chem. 273:20456-20462.

24. Qiu, S., et al. 1998. Metabolism of lipoproteins containing apolipoprotein B in hepatic lipase-deficient mice. J. Lipid Res. 39:1661-1668. 